\title{
ELECTRONIC MAIL
}

\author{
Chris Benn CRBQUK.AC.RGO.sTAR on JANET
}

Royal Greenwich Observatory, Herstmonceux, East Sussex, BN27 1RP, United Kingdom

\section{I - Introduction}

Astronomers have always had a special need for rapid communication over large distances, partly because of their interest in experiments requiring long baselines. Examples: Eratosthenes' measurement of the circumference of the Earth; determination of longitude at sea; solar-eclipse tests of general relativity; very-long-baseline interferometry. Of these, at least the second has prompted innovations in communications techniques (beginning with the Greenwich time ball).

More recently, the pressing need to travel widely for clean air and aether, and the international flavour of the resulting collaborations, has given astronomers particular incentive to explore the latest communications innovation: electronic mail.

Electronic mail travels across data-communications networks which straddle the planet like overlapping spiders' webs. Most large computers are connected to them. Electronic mail is cheap, quick and convenient, and for text transmission offers an attractive alternative to other communications media. In particular, it allows astronomers separated by great distances to interact with some of the informality with which they might chat over coffee.

\section{II - The networks}

To send an electronic-mail message, it is necessary to know the address (username and computer name) of the intended recipient, and the name of the network to which the computer is connected. The networks most commonly used by astronomers are listed below:

Network

ACSNET
BITNET/EARN
INFNET
INTERNET
JANET
SPAN
UUCP

Area

Australia

North America / Europe Italy (academic DECnet)

USA (ARPA net etc.) UK academic network USA / Europe space network Worldwide (UNIX)

Worldwide
Example electronic-mail address

\begin{tabular}{|c|c|}
\hline FREDQAAOEPP.OZ.AU & (Fred at AAO) \\
\hline FREDOSEMAX51 & (Fred at Uppsala) \\
\hline ASTB01::FRED & (Fred at Bologna) \\
\hline FREDQASTRO.AS.UTEXAS.ED & U (Fred at Texas) \\
\hline FRED OUK.AC.RGO.STAR & (Fred at RGO) \\
\hline SCIVAX::FRED & t STScI) \\
\hline MCVAX!ENEA!AS & (Fred at Lund) \\
\hline 28621001::FRED & (Fred at CSIRO) \\
\hline
\end{tabular}

There are many other national networks, for example FUNET in Finland, JUNET in Japan and STARLINK in the UK. Links with the Soviet Union and China are imminent, and many developing countries are becoming accessible.

The gateways between the networks allow millions of individual users to send electronic messages to one another's terminals. However, after tacking on instructions for propelling a message through the gateways, an electronic-mail address can become a veritable jungle of acronyms and punctuation marks, and the ugly syntax may intimidate novice users. 


\section{III - The RGO guide to electronic mail}

To make network mail more accessible to the astronomical community, I have compiled, in collaboration with Ralph Martin of RGO, a short guide to help users master the idiosyncracies of the subject, and a directory of electronic-mail addresses for astronomers. The directory lists electronicmail addresses (username, computer name and network name) for $\approx 3000$ astronomers worldwide and electronic-mail, telephone, telex, fax and postal addresses for $\approx 200$ observatories. Paper and electronic copies of the guide and directory are available from the author. Readers are encouraged to submit requests by electronic mail.

Updates of the directory are distributed every 6 months, by electronic mail, to representatives at each of 150 institutions worldwide. These representatives are responsible for local distribution and for providing update information for the next edition. Readers who find that their entries in the directory are incorrect, or who can't find them at all, should contact the author.

\section{IV - Pitfalls and problems}

The networks are now quite reliable, with few black holes. If a message is obstructed en route, it will usually be bounced back to the sender with a helpful explanation of what happened. The commonest problem, affecting maybe $5 \%$ of transmitted messages, is that the computer at the receiving end is comatose. The machine may for example have been switched off for the weekend, or it may be down for maintenance. Some mailers will keep trying to get through, and will only give up after a set number of hours have elapsed.

Machine names and routings still change, but for astronomers, who tend to be itinerant, electronic-mail addresses are nearly as durable as postal ones (perhaps journal editors should encourage inclusion of authors' electronic-mail addresses at the head of papers).

Size limits, typically 100000 bytes (characters), are often encountered on the UUCP network. They can be evaded by chopping the message into chunks and sending it piecemeal.

Sometimes a message is rejected by the destination computer, usually because: the username has ceased to exist; or the user has inadequate discspace in which to store the message; or the user has accidentally protected his mail file against writing by other users.

A message which negotiates all these hurdles will arrive, but it may have been trimmed (often to 80 characters per line), and/or corrupted. Special characters such as ' $\{$ ' and ' $\}$ ' are particularly susceptible to mis-translation when being passed between computers using different character codes (e.g. ASCII, EBCDIC). Much to the distress of $T_{E} X$ users, beloved of such esoteric characters, the corruption is usually irreversible. A pragmatic solution is global replacement of all special characters by unusual strings befor mailing, the recipient of the message being required to carry out the reverse edit. For example, ' $\{$ ' might be represented by ' $;:($ ', and ' $\}$ ' by ' $;:)$ '.

Electronic mail is not secure. Incoming mail is stored in ordinary disk files, and these can often be read by other users of the computer. Only encryption guarantees privacy. A codeword-driven encryption/decryption program is available from the author.

Speed of transmission varies from network to network and with geographical distance. Most messages arrive within seconds or minutes, but inter-network messages can take hours. In fact, the latter often achieve about the same mean ground speed as the hilltop-to-hilltop bonfire method used in ancient Mesopotamia, albeit with a higher bit rate. 


\section{V - Naming conventions}

Most computer usernames are constructed from initials (e.g. LDV), from surname (e.g. DAVINCI) or from first initial + surname (e.g. LDAVINCI). The last of these conventions has been adopted by many large observatories, including NOAO and NRAO. It generates usernames which are easy to guess, but which are unlikely to be duplicated. In continental Europe, matters are slightly complicated by the use of accents, which are sometimes translated into extra vowels in the username.

Many sites support institutional usernames, such as DIRECTOR, INFORMATION, LIBRARY and POST. MASTER. Don Wells of NRAO has suggested that standard usernames for observers and operators at telescopes might be constructed, e.g. as OBSx and OPRx, where $\mathrm{x}$ is the abbreviated name of the telescope.

Great confusion is caused by mixing the characters $0,0,1$ and 1 in the names assigned to users or computers: They are difficult to distinguish, particularly when handwritten. The inelegant EARN computer name DGAES051, for example, might easily be read as DGAES051, unless the reader spots the embedded initials of the European Southern Observatory. An even worse source of confusion is the Scandinavian letter $\emptyset$, which resembles the symbol used by programmers to represent a zero.

\section{VI - The future}

Electronic mail is a novel technology. I think that it may prove as stimulating to astronomy as, say, the advent of cheap air travel, or the invention of a new type of detector. It is revolutionising communications between astronomers, and will doubtless take over many of the roles currently occupied by post, telephone, telex, fax, conferences and even publication. I would like to highlight two examples: software exchange, and dissemination of news and ideas.

Much of the software which an astronomer needs already exists, but it's usually easier to write one's own than to obtain a tape copy of somebody else's. With electronic mail, the transmission of computer programs becomes cheap, quick and easy. No index of computer programs useful to astronomers yet exists, but one is being prepared by the software working group of Commission 5 .

Electronic mail is an ideal medium for the transmission of news. Issues of an electronic journal containing electronically-submitted news items about telescopes, satellite launches, conferences, new software etc. could be compiled and transmitted quickly and at very little cost, to nearly all the observatories in the West. Such a journal could not include sophisticated figures, but it could offer a speedier and more comprehensive coverage than the current confetti of newsletters, and it would be easier to search for keywords.

An electronic journal might also be a suitable medium for the dissemination and discussion of ideas. At present, this takes place mainly through the literature (on a long timescale), at conferences (at considerable cost), or through personal contact (within a limited circle). Electronic dissemination might help to scatter half-baked thoughts on fertile ground, and thus bring to fruition ideas which would otherwise have perished.

Electronic mail is already well-established in the business and academic worlds, and is poised to invade the home. Telecommunications companies are predicting integrated fibre-optics networks for TV, telephone and electronic mail to most homes, and are anticipating the availability of associated facilities for video-mailing, language-translation and even speech-recognition within a few years. In France, a substantial fraction of homes are already equipped with government-supplied computer terminals. Electronic mail is obviously here to stay, perhaps as a harbinger of the global electronic village envisioned by Arthur C. Clarke and others. 
SEARCHING FOR ASTRONOMICAL INFORMATION

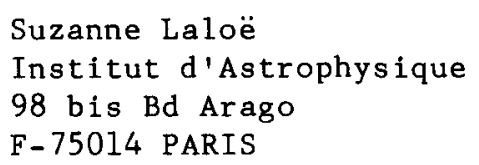

Searching for astronomical information can mean trying to answer such diverse questions as :

- diameter of the earth ?

- objects with a redshift $>4$ ?

- ubv data for stars within 3 degres of

alpha $=1045.2 ;$ delta $=+1251$

- very recent paper by XXXX ?

What are the tools we have? Libraries and data centers, which have to help us get any information by taking the greatest care in the :

- classification

- lists of terms

they use, and by communicating with each other to have the most comprehensive service.

In Session 2, we heard of what is available to the astronomical community (including the latest products such as Astronomy and Astrophysics Monthly Index and SIMBAD, as well as the more "classical" like Astronomy and Astrophysics Abstracts, INSPEC,...) and what still needs to be done to improve retrieval of information (construction of a thesaurus, union lists of serials, wider use of electronic communications).

But I think we should always keep in mind that often, a simple phone call to a colleague, a visit to the scientist next door, an electronic message to "someone who might know someone who knows"... will provide more relevant answers than the most sophisticated tools.

So, never forget the simplest and cheapest way to get good information : TALK to other people! 